\title{
Microglial NLRP3 inflammasome activation mediates IL-1 $\beta$ release and contributes to central sensitization in a recurrent nitroglycerin-induced migraine model
}

Wei He' , Ting Long ${ }^{1}$, Qi Pan ${ }^{1}$, Shanshan Zhang ${ }^{1}$, Yixin Zhang ${ }^{1}$, Dunke Zhang ${ }^{2}$, Guangcheng Qin $^{2}$, Lixue Chen ${ }^{2}$ and Jiying Zhou ${ }^{1 *}$ (1)

\begin{abstract}
Background: Central sensitization is an important mechanism of chronic migraine (CM) and is related to the inflammatory response of microglia. The NOD-like receptor protein 3 (NLRP3) inflammasome may regulate the inflammatory process of microglia in several neurological diseases, but its role in CM is largely unknown. Therefore, the aim of this study was to identify the precise role of microglial NLRP3 in CM.

Methods: An experimental CM mouse model was established by repeated intraperitoneal (i.p) injection with nitroglycerin (NTG). We evaluated the expression levels of NLRP3 and its downstream interleukin (IL)-1 $\beta$ protein in the trigeminal nucleus caudalis (TNC; which is a central area relevant to migraine pain) at different time points. To further examine the effects of the NLRP3 inflammasome pathway on central sensitization of CM, we examined MCC950, an NLRP3 inflammasome-specific inhibitor, and IL-1ra, an IL-1 $\beta$ antagonist, whether altered NTG-induced mechanical hyperalgesia of the periorbital area and hind paw. The effect of MCC950 and IL-1ra on c-Fos, phosphorylated extracellular signal-regulated kinase (p-ERK) and calcitonin gene-related peptide (CGRP) expression in the TNC were also analyzed. The cell localization of NLRP3 and IL-1 $\beta$ in the TNC was evaluated by immunofluorescence staining.

Results: Repeated NTG administration induced acute and chronic mechanical hyperalgesia and increased expression of NLRP3 and IL-1 $\beta$. Blockade of NLRP3 or IL-1 $\beta$ reduced NTG-induced hyperalgesia, and this effect was accompanied by a significant inhibition of the NTG-induced increase in p-ERK, c-Fos and CGRP levels in the TNC. Immunofluorescence staining revealed that NLRP3 and IL-1 $\beta$ were mainly expressed in microglia in the TNC, and the IL-1 $\beta$ receptor, IL-1R, was mainly expressed in neurons in the TNC.

Conclusions: These results indicate that NLRP3 activation in the TNC participates in the microglial-neuronal signal by mediating the inflammatory response. This process contributes to the central sensitization observed in CM.
\end{abstract}

Keywords: Chronic migraine, Nod-like receptor protein 3 inflammasome, Interleukin-1 $\beta$, Central sensitization

\footnotetext{
* Correspondence: zheadache@163.com

'Department of Neurology, The First Affiliated Hospital of Chongqing

Medical University, 1st You Yi Road, Yu Zhong District, Chongqing 400016,

People's Republic of China

Full list of author information is available at the end of the article
}

(c) The Author(s). 2019 Open Access This article is distributed under the terms of the Creative Commons Attribution 4.0 International License (http://creativecommons.org/licenses/by/4.0/), which permits unrestricted use, distribution, and reproduction in any medium, provided you give appropriate credit to the original author(s) and the source, provide a link to the Creative Commons license, and indicate if changes were made. The Creative Commons Public Domain Dedication waiver (http://creativecommons.org/publicdomain/zero/1.0/) applies to the data made available in this article, unless otherwise stated. 


\section{Introduction}

Chronic migraine $(\mathrm{CM})$ is a complex neurological disease which usually progresses from episodic migraine [1], and the global prevalence is $0.5-1 \%$ [2]. Due to limited treatment options and effects, more than half of patients with CM reported dissatisfaction with their treatments [3]. A better understanding of the molecular mechanism of $\mathrm{CM}$ will provide new therapeutic approaches.

The current theory suggests that CM may be due to the chronic sensitization of central pain pathways caused by repeated migraine attacks [4]. Among these pathways, the activation of the trigeminal nucleus caudalis (TNC) area is an important manifestation of central sensitization [4]. In addition to neurons, microglia and the inflammatory factors they secrete are also involved in the regulation of central sensitization $[5,6]$. This involvement has been extensively demonstrated in other pain models [7]. In recent years, the role of microglia in migraine has also been preliminarily understood [8]. According to our previous study, microglia were associated with central sensitization in CM [9]. However, the specific intracellular mechanism is unclear, which is the current focus of our research.

Based on accumulating evidence, IL-1 $\beta$ is involved in migraine pathology. For example, during a migraine attack and the interictal periods, IL-1 $\beta$ markedly increases in peripheral blood [10]. In the trigeminal ganglion (TG), IL-1 $\beta$ mediates the pro-inflammatory process, contributes to the activation of the trigeminal satellite cells and promotes cross excitation of satellite glial cells and neurons [11]. However, these studies focused on the peripheral area. In the central area of the trigeminovascular pathway, especially the TNC, which is the important area of the development and maintenance of CM, the mechanism of action of IL-1 $\beta$ is largely unknown.

Considerable evidence from animal models of neuroinflammation suggests that IL-1 $\beta$ of the central nervous system (CNS) is mainly from microglia [12]. The maturation of IL-1 $\beta$ has a unique molecular mechanism in a kind of intracellular multiprotein complex, the NOD-like receptor protein3 (NLRP3) inflammasome. The NLRP3 inflammasome is an innate immune complex that mediates the activation of caspase-1, which in turn cleaves pro-IL-1 $\beta$ to form mature IL-1 $\beta$ [13]. This function is crucial for the regulation of neuroinflammation mediated by microglia.

The NLRP3 inflammasome has been associated with several inflammatory disorders of the CNS, such as depression [14], spontaneous intracerebral haemorrhage [15] and multiple sclerosis [16]. However, the role of NLRP3 in $\mathrm{CM}$ has not been reported. Notably, the conditions for NLRP3 activation, such as the high concentrations of extracellular $\mathrm{K}+$ ions $[17,18]$, increased peroxynitrite, mitochondrial dysfunction and the generation of reactive oxygen species (ROS), usually underlie the generation of a migraine [19-23].
Therefore, investigating the role of the NLRP3 inflammasome in $\mathrm{CM}$-associated pathology and functional outcomes is intriguing. We hypothesized that the NLRP3 inflammasome has the potential to induce IL- $1 \beta$-related neuroinflammation in $\mathrm{CM}$. Continuous inflammatory processes contribute to central sensitization.

In this study, we aimed to investigate the role of the NLRP3/IL-1 $\beta$ pathway in nitroglycerin (NTG)-induced hyperalgesia and its underlying mechanism in the TNC. We expect to identify an effective therapeutic approach for CM.

\section{Materials and methods \\ Animals}

The subjects were male C57BL6/J mice, weighing 18-25 $\mathrm{g}$ and aged 8-12 weeks. The mice were provided by the Experimental Animal Center of Chongqing Medical University (Chongqing, China). Mice were maintained at a temperature of $22 \pm 2{ }^{\circ} \mathrm{C}$ with a humidity of $50 \pm 5 \%$ on a 12-h light-dark cycle, and food and water were freely available. All procedures involving the use of animals were approved by the Animal Care and Use Committee at Chongqing Medical University in China. Animal experiments were also carried out in accordance with the ethical guidelines of the International Association for the Study of Pain for investigations of experimental pain in conscious animals [24]. All experiments were performed in a blinded manner, and mice were habituated for 1 week before any experimental procedures were initiated. Animals were weighed daily during treatment, and no adverse effects of treatment were observed.

\section{Establishment of a chronic migraine model}

The procedures listed below were performed as described in the report by Pradhan et al. [25]. We established a CM model by repeated intraperitoneal (i.p) injection with NTG. NTG was purchased from the First Affiliated Hospital of Chongqing Medical University (Chinese Drug Approval Number: H11020289; 5.0 mg/ml). No significant differences in the mechanical thresholds were observed between animals injected with $0.9 \%$ saline and the NTG solvent $(6 \%$ propylene glycol, $6 \%$ alcohol, and $0.9 \%$ saline) [25]; therefore, the vehicle control used in the experiments was $0.9 \%$ saline. NTG was freshly diluted in $0.9 \%$ saline to a dose of $10 \mathrm{mg} / \mathrm{kg}$. All injections were administered at a volume of $10 \mathrm{ml} / \mathrm{kg}$. The model group received an NTG injection every other day for a total of five times over 9 days (in the chronic treatment experiment, the injections were performed five times, on days $3,5,7,9$ and 11).

\section{Intracerebroventricular cannulation}

Intracerebroventricular (I.C.V.) cannulation was performed as described previously [26]. Mice were anaesthetized with $10 \%$ chloral hydrate $(4 \mathrm{ml} / \mathrm{kg}$, i.p.) and positioned in a stereotaxic apparatus (ST-51603; 30 Stoelting Co., Chicago, 
IL, USA). A 26-gauge stainless steel cannula was placed in the left lateral cerebral ventricle at predetermined coordinates (lateral $1.6 \mathrm{~mm}$ and anteroposterior $1 \mathrm{~mm}$ to bregma and horizontal $2 \mathrm{~mm}$ from the dura mater) [27]. The cannula was secured using dental acrylic. Mice were provided a minimum of 5 days to recover before any treatment or behavioural test.

\section{Treatment drug administration}

Experiment 2 used the NLRP3 inhibitor MCC950 (Thermo, Houston, TX, USA), which has a specific and strong inhibitory effect on NLRP3 [28, 29]. After i.p. injection, MCC950 can pass through the blood-brain barrier to act on the central area [29]. MCC950 was dissolved in sterile phosphate-buffered saline (PBS) at a concentration of $10 \mathrm{mg} / \mathrm{ml}$, and each mouse was given a dose of $10 \mathrm{mg} / \mathrm{kg}$ with i.p. injection once a day for 11 days. The doses for MCC950 used in the experiment were based on previous studies [29]. Sterile PBS of an equal volume was used as the control.

Experiment 3 used an IL-1 receptor antagonist, IL-1ra (PeproTech, Rocky Hill, NJ, USA). IL-1ra was dissolved in sterile PBS at a concentration of $2 \mu \mathrm{g} / \mu \mathrm{l}$, and each mouse was given a $4 \mu \mathrm{g}$ I.C.V. injection once a day for 11 days. The doses used in this experiment were based on previous studies [27]. Sterile PBS of an equal volume was used as the control.

\section{Sensory sensitivity testing}

We used the von Frey test to examine the mechanical sensitivity, the threshold for responses to punctuate mechanical stimuli (mechanical hyperalgesia). The tests used the electronic von Frey device (Electrovonfrey, IITC Inc., Woodland Hills, CA, USA) that automatically recorded thresholds after stimulation. The investigators were blinded to the experimental groups. All the mice were habituated to the test chamber before testing began.

Before testing the periorbital sensitivity, the mice were placed into a $9-\mathrm{cm}$-long restraining glass cylinder such that only the head poked out. The restrainer allowed head and forepaw movements but prevented the animals from turning in the cylinder. Then, following application of an electronic von Frey monofilament to the periorbital area of the face over the rostral portion of the eye, a positive response was defined as when the mouse stroked its face with the ipsilateral forepaw, quickly retracted its head from the stimulus, or vocalized. To test the hind paw sensitivity, we placed mice in wire mesh boxes, applying an electronic von Frey monofilament to the plantar surface of the animal hind paw from the underside of the mesh stand. A positive response with hind paw was defined as withdrawal, shaking, or licking of the paw in response to stimulation. Each site was tested at least three times with an interval of at least $1 \mathrm{~min}$.

In experiment 1 , days $1,3,5,7$ and 9 were testing days. Mice were tested for mechanical thresholds before injection (baseline responses) and $2 \mathrm{~h}$ after injection with NTG or vehicle.

In experiments 2 and 3, days 3, 5, 7, 9 and 11 were testing days. The treatment of the mice on testing days was as follows: all mice were first tested for baseline mechanical thresholds, followed by injection of preventative therapy (MCC950 or IL-1ra) or their respective vehicles; then administration of NTG after 30-45 min; and finally, at 2-h post-NTG injection, mice were tested again for mechanical responses.

An additional figure file shows this procedure in more detail [see Additional file 1].

\section{Quantitative real-time reverse trancriptase polymerase chain reaction (qRT-PCR)}

Mice were anesthetized and decapitated $24 \mathrm{~h}$ after the last NTG or saline injection, and the TNC tissues were obtained and immediately stored in liquid nitrogen until analysis. Total RNA was purified using an RNAiso Plus reagent (Takara, Tokyo, Japan), and yield and purity were assessed spectrophotometrically with a NanoDrop spectrophotometer (Thermo, Waltham, MA, USA). cDNAs were synthesized using a PrimeScript ${ }^{\mathrm{Tu}}$ RT Reagent Kit (Takara). Gene-specific primers were obtained from TSINGKE Biological Technology (Chengdu, China), and specificity was tested by basic local alignment search tool (BLAST). qRT-PCR was performed with SYBR ${ }^{\circ}$ Premix Ex Taq ${ }^{\text {Tw }}$ II (Takara) using a CFX96 Touch thermocycler (Bio-Rad, Hercules, CA, USA). All final expression data were expressed as a target/reference ratio in experimental samples and normalized to the target/reference ratio of the calibrator. At the end of each run, melting curve analysis was performed to verify the single-product detection of the primers. All fluorescence data were processed by a post-PCR data analysis software program, and relative gene expression was normalized to the internal reference glyceraldehyde 3 -phosphate dehydrogenase (GAPDH) using the $2^{-\Delta \Delta C T}$ method [30]. Sequence-specific primers for NLRP3, IL- $1 \beta$ and GADPH are shown in Table 1.

\section{Western blot analysis}

For experiment 1 to detect the NLRP3 and IL-1 $\beta$ proteins, TNCs were collected $24 \mathrm{~h}$ after different NTG injections. For experiments 2 and 3 , TNCs were collected $24 \mathrm{~h}$ after the last NTG/saline injection to detect the levels of NLRP3, IL-1 $\beta$ and calcitonin gene-related peptide (CGRP) proteins, and TNCs were collected $2 \mathrm{~h}$ after the last NTG/ saline injection to detect levels of the phosphorylated extracellular signal-regulated kinase (p-ERK) and c-Fos 
Table 1 Sequences of primers used

\begin{tabular}{lll}
\hline Gene & Forward primer & Reverse primer \\
\hline NLRP3 & CCATCAATGCTGCTTCGACA & GAGCTCAGAACCAATGCGAG \\
IL-1 $\beta$ & GTGAAATGCCACCTTITGACAGTGA & GAAGGTCCACGGGAAAGACAC \\
GAPDH & AGACAGCCGCATCTTCTTGT & TGATGGCAACAATGTCCACT \\
\hline
\end{tabular}

proteins. Mice were anesthetized and decapitated, and fresh samples were homogenized in buffer containing protease and phosphatase inhibitor cocktails (Beyotime, Shanghai, China). Protein concentrations were determined using a bicinchoninic acid (BCA) Protein Assay kit (Beyotime). Equal amounts of protein samples $(40 \mu \mathrm{g})$ were loaded on sodium dodecyl sulfate polyacrylamide gel electrophoresis (SDS PAGE) gels, electrophoresed and transferred to a nitrocellulose membrane. Membranes were blocked with 5\% non-fat milk in Tris-buffered saline (TBS) containing $0.1 \%$ Tween 20 for $2 \mathrm{~h}$ at room temperature and incubated with each antibody at $4{ }^{\circ} \mathrm{C}$ overnight. The primary antibodies were anti-NLRP3, anti-IL-1 $\beta$, anti-c-Fos, anti-p-ERK and anti-
CGRP antibodies [see Table 2]. An anti- $\beta$-actin antibody was used as the loading control. Then, horseradish peroxidase conjugated secondary antibodies were applied for $2 \mathrm{~h}$ at room temperature. The membranes were probed with a BeyoECL Plus kit (Beyotime). The ImageJ analysis system (Fusion, Germany) was used to quantify the specific bands.

\section{Immunofluorescence (IF) staining}

For ionized calcium binding adaptor molecule 1 (Iba-1), NLRP3, IL-1 $\beta$ and CGRP detection, tissues were collected $24 \mathrm{~h}$ after the last NTG or vehicle injection. For p-ERK detection, tissues were collected at $2 \mathrm{~h}$ after the last NTG or vehicle injection. Mice were anesthetized

Table 2 Antibodies used in Western blot analysis, immunofluorescence staining and immunohistochemistry

\begin{tabular}{|c|c|c|c|c|}
\hline Antibody & Manufacturer & Catalogue number & Host & Dilution \\
\hline \multicolumn{5}{|l|}{ For Western blot analysis } \\
\hline NLRP3 & Abcam, UK & ab205680 & Rat & $1: 1000$ \\
\hline $\operatorname{IL}-1 \beta$ & Santa Cruz, USA & sc- $-515,598$ & Mouse & $1: 3000$ \\
\hline c-Fos & Santa Cruz, USA & sc- -447 & Mouse & $1: 1200$ \\
\hline $\mathrm{p}$-ERK & Cell Signaling, USA & 5726 & Mouse & $1: 1000$ \\
\hline CGRP & Abcam, UK & ab10062 & Mouse & $1: 2000$ \\
\hline$\beta$-actin & Proteintech, China & 20,536-1-AP & Rabbit & $1: 9000$ \\
\hline Anti-rabbit lgG (HRP) & ZSGB-BIO, China & ZB-2301 & Goat & $1: 5000$ \\
\hline Anti-mouse lgG (HRP) & ZSGB-BIO, China & ZB-2305 & Goat & $1: 5000$ \\
\hline Anti-rat lgG (HRP) & ZSGB-BIO, China & ZB-2307 & Goat & 1:5000 \\
\hline \multicolumn{5}{|l|}{ For immunofluorescence staining } \\
\hline NLRP3 & Biorbyt & orb101128 & Rabbit & $1: 400$ \\
\hline CGRP & Santa Cruz, USA & sc- $-57,053$ & Mouse & $1: 100$ \\
\hline $\mathrm{IL}-1 \beta$ & Santa Cruz, USA & sc-515,598 & Mouse & $1: 50$ \\
\hline IL-1R & Abcam, UK & ab124962 & Rabbit & $1: 200$ \\
\hline Iba-1 & Santa Cruz, USA & sc- $-32,725$ & Mouse & $1: 200$ \\
\hline Iba-1 & Wako, Japan & $019-19,741$ & Rabbit & $1: 500$ \\
\hline GFAP & Abcam, UK & ab10062 & Mouse & $1: 100$ \\
\hline NeuN & Abcam, UK & ab104224 & Mouse & $1: 500$ \\
\hline Alexa Fluor 488 goat anti-rabbit lgG & Beyotime, China & A0423 & Goat & $1: 400$ \\
\hline Alexa Fluor 488 goat anti-mouse lgG & Beyotime, China & A0428 & Goat & $1: 400$ \\
\hline Cy3 goat anti-rabbit lgG & Beyotime, China & A0516 & Goat & $1: 400$ \\
\hline Cy3 goat anti-mouse lgG & Beyotime, China & A0521 & Goat & $1: 400$ \\
\hline \multicolumn{5}{|l|}{ For immunohistochemistry } \\
\hline c-Fos & Abcam, USA & ab208942 & Mouse & $1: 1000$ \\
\hline
\end{tabular}


and perfused intracardially with $15 \mathrm{ml}$ ice-cold $0.1 \mathrm{M}$ PBS (pH 7.2) and then with $50 \mathrm{ml}$ ice-cold $4 \%$ paraformaldehyde (PFA)/0.1 M PBS. Whole brains, including TNCs, were post-fixed overnight with $4 \%$ PFA/0.1 M PBS at $4{ }^{\circ} \mathrm{C}$. The tissues were cryoprotected in $30 \%$ sucrose/0.1 M PBS for $24-36 \mathrm{~h}$ or until the tissue sank. Brain tissues were flash frozen in 2-methyl butane on dry ice, and coronal sections of the TNC were sliced at $10 \mathrm{~mm}$. All sections within bregma at $-7.47 \mathrm{~mm}$ to $-8.24 \mathrm{~mm}$ were collected [31].

Immunofluorescence and double immunostaining were performed on cryofixed sections. The sections were incubated at $4{ }^{\circ} \mathrm{C}$ overnight with primary antibodies, as listed in Table 2, antibodies against NLRP3, CGRP, IL-1 $\beta$, and IL-1R and antibodies against Iba-1, glial fibrillary acidic protein (GFAP) and neuronal nuclei (NeuN) (used to mark microglia, astrocytes and neurons, respectively). We used 4',6-diamidino-2-phenylindole (DAPI) for nuclear staining. Alexa Fluor 488 and Cy3-labelled goat anti-mouse or goat anti-rabbit immunoglobulin (IgG) were used as secondary antibodies (see Table 2). Slides containing the negative controls were incubated with TBS instead of primary antibodies, which resulted in no positive labeling of the tissues.

\section{Immunofluorescence imaging data analysis}

The TNC area was determined based on morphological appearance under a light microscope using the Mouse Brain Atlas as a reference [31]. Sections were viewed under a confocal laser scanning fluorescence microscope (ZEISS, Oberkochen, Germany). Laminae I-V of the TNC was determined manually as the area of interest using a rectangular tool. A significant difference in the area of the TNC was not observed among the experimental groups. Images were analyzed by using Image-Pro Plus 6.2 software (Bethesda, MD, USA). The ratio of the cross-sectional area immunoreactive for Iba- 1 and p-ERK were calculated in the TNC at $\times 20$ magnification. The fluorescence intensity of CGRP was presented by mean optical density (OD) in the TNC at $\times 10$ magnification. TNCs in both hemispheres from six sections per animal ( $n=6$ mice/ group) were included in the analysis. All evaluations were performed by an observer who was blinded to the experimental groups.

\section{Immunohistochemistry $(\mathrm{IH})$ staining}

For c-Fos detection, sections were incubated with $0.3 \%$ $\mathrm{H}_{2} \mathrm{O}_{2}$ for $30 \mathrm{~min}$ and blocked with $10 \%$ normal goat serum for $2 \mathrm{~h}$. These sections were incubated overnight at $4{ }^{\circ} \mathrm{C}$ with a primary antibody against c-Fos (Table 2) and then incubated with a secondary antibody solution (biotinylated goat anti-mouse antibody; ZSGB-BIO,
Beijing, China) for $2 \mathrm{~h}$ at room temperature. Next, sections were incubated in horseradish enzyme-labelled streptomyces lecithin reagent (ZSGB-BIO) for $1 \mathrm{~h}$ at room temperature. Nickel-enhanced diaminobenzidine (DAB) was used to visualize primary antibody staining. Sections were mounted, dried and dehydrated.

\section{Immunohistochemistry imaging data analysis}

The TNC area of interest in the images of each section was determined as previously described. Images were observed under a ZEISS Axio Imager A2 microscope using a $\times 20$ objective lens. Images were analyzed using Image-Pro Plus 6.0 software. The expression of c-Fos was blindly examined and quantified by counting c-Fos-positive cells from averaging six TNC sections per mouse ( $n=6$ mice/group).

\section{Statistical analyses}

All data are presented as the means \pm standard deviations (SDs). All statistical analyses and graphs were performed using GraphPad Prism version 6.0 (GraphPad Software Inc., San Diego, CA, USA). Statistical analysis of the mechanical threshold data was performed using two-way repeated measures analysis of variance (ANOVA), with drug and time as factors. Western blot data were analyzed by one-way ANOVA followed by Tukey's multiple comparison test. For IF, immunohistochemical and qRT-PCR experiments, data were analyzed using unpaired $t$ test (if the variances were significantly different, unpaired $t$ test with Welch's correction was used.) In all statistical comparisons, a $P$ value $<0.05$ was considered significant.

\section{Experimental design}

An additional figure file shows this process in more detail [see Additional file 1].

\section{Experiment 1}

This experiment was performed to confirm whether repeated NTG stimulation could cause migraine-associated hyperalgesia in mice and form a central sensitization state. This experiment was also performed to verify whether NLRP3 and its related pathways were involved in this process.

Animals were given NTG or saline injections every other day (on days 1, 3, 5, 7 and 9) and tested of basal mechanical responses and NTG post-treatment responses (details in the "Materials and methods" section). The expression levels of the NLRP3 and IL-1 $\beta$ proteins were detected by Western blotting $24 \mathrm{~h}$ after the each NTG or saline injections. Two hours after the last NTG or saline injection, we tested c-Fos protein levels by IH and tested p-ERK levels by IF. At $24 \mathrm{~h}$ after the last NTG or saline injection, we tested NLRP3 and IL-1 $\beta$ 
mRNA levels by qRT-PCR and tested Iba-1 (microglial maker) and CGRP protein levels in the TNC by IF, as well as double IF for Iba-1, NLRP3 and IL-1 $\beta$.

\section{Experiment 2}

This experiment was performed to test the preventative effect of NLRP3 inhibition on NTG-induced migraine-associated pain.

A specific small molecule inhibitor of the NLRP3 inflammasome, MCC950, was used. The following groups were set up for the experiment: saline group, NTG group, NTG + MCC950 group and NTG+vehicle (VEH) group. For the saline or NTG group, mice were given saline or NTG injections on days 3, 5, 7, 9 and 11. For the NTG+MCC950 or NTG+VEH group, mice were injected once daily with MCC950 (10 mg/kg, i.p.) or VEH (equal volumes of PBS) for 11 days. On days 3, 5, 7, 9 and 11, mice were injected with NTG after being injected MCC950 or VEH. All mice were tested for basal mechanical responses and NTG post-treatment responses (details in the "Materials and methods" section). The levels of the IL- $1 \beta$ and CGRP proteins were detected by Western blotting $24 \mathrm{~h}$ after the NTG injection on day 11. The levels of the c-Fos and p-ERK proteins were detected by Western blotting $2 \mathrm{~h}$ after NTG injection on day 11.

\section{Experiment 3}

This experiment was performed to test the preventative effect of IL-1 $\beta$ blockage on NTG-induced migraine-associated pain.

An IL-1 receptor antagonist, IL-1ra, was used to block IL-1 $\beta$. The following groups were established for the experiment: saline group, NTG group, NTG + IL-1ra group and NTG+VEH group. For the saline or NTG group, mice were given saline or NTG injections on days $3,5,7,9$ and 11 . For the NTG+IL-1ra or NTG+VEH group, mice underwent I.C.V. cannulation and were I.C.V. injected with IL-1ra $(4 \mu \mathrm{g} /$ mouse/day) or VEH (equal volumes of sterile PBS) once daily for 11 days. On days $3,5,7,9$ and 11 , mice were injected with NTG after being injected with IL-1ra or VEH. All mice were tested for basal mechanical responses and NTG post-treatment responses (details in the "Materials and methods" section). The levels of NLRP3 and CGRP proteins were detected by Western blotting $24 \mathrm{~h}$ after the NTG injection on day 11. The levels of c-Fos and p-ERK proteins were detected by Western blotting $2 \mathrm{~h}$ after NTG injection on day 11. The tissue for double IF analysis of the IL-1 receptor (IL-1R) with NeuN, Iba-1 and GFAP was collected $24 \mathrm{~h}$ after the NTG injection on day 11.

\section{Results}

Repeated NTG stimulation induced mechanical hyperalgesia

For experiment 1 , mice were administered NTG or saline every other day (Fig. 1a). The baseline mechanical thresholds before administration were not significantly different between the two groups ( $p>0.05$; Fig. 1b, c date on the first day). However, the baseline mechanical thresholds of the hind paw and periorbital area were significantly decreased in the NTG group compared with those in the control (saline) group after the first injection $(p<0.001$; Fig. 1b, c). Repeated administration of NTG maintained the reduction in the baseline mechanical threshold, which was most obvious on days 7 and 9 . In addition, compared to the saline group, each NTG administration induced significant post-treatment (acute) hyperalgesia $(p<0.001$; Fig. 1d, e).

\section{Repeated NTG stimulation increased expression of the c- Fos, $p$-ERK and CGRP in the TNC}

The activation of c-Fos and the phosphorylation of ERK have been used as a molecular reliable marker for neurons activation and central sensitization [32]. Our study have shown that repeated NTG stimulation induced significant increases in the protein expression levels of the p-ERK and c-Fos in the TNC (Fig. 2b, c, f, g). Based on these results, NTG induced acute hyperalgesia, which may be associated with the activation of c-Fos and p-ERK in headache-related brain regions (the TNC regulates craniofacial pain). CGRP is considered an endogenous migraine generator and plays a critical role in the initiation and maintenance of a migraine [33]. Repeated NTG stimulation reliably induced a significant basal hypersensitivity. To determine whether the basal hypersensitivity induced by repeated NTG administration corresponded to increased CGRP expression, we determined CGRP expression $24 \mathrm{~h}$ after the final administration of NTG in the TNC and observed significantly increased (Fig. 2d, e).

\section{Repeated NTG stimulation induced NLRP3 inflammasome activation}

Consistent with our previous published study [9], the number of Iba-1-marked microglia cells was increased in the TNC after repeated administration of NTG. An additional figure file shows this finding in more detail [see Additional file 2]. We speculated that microglial activation correlated with the NTG-induced hyperalgesia. Hence, we investigated the role of the relevant pathways of microglia, such as the NLRP3 inflammasome pathway in CM-associated pathology and function.

Our data shows that compared to repeated saline administration mice (control group), repeated NTG administration increased the mRNA and protein 


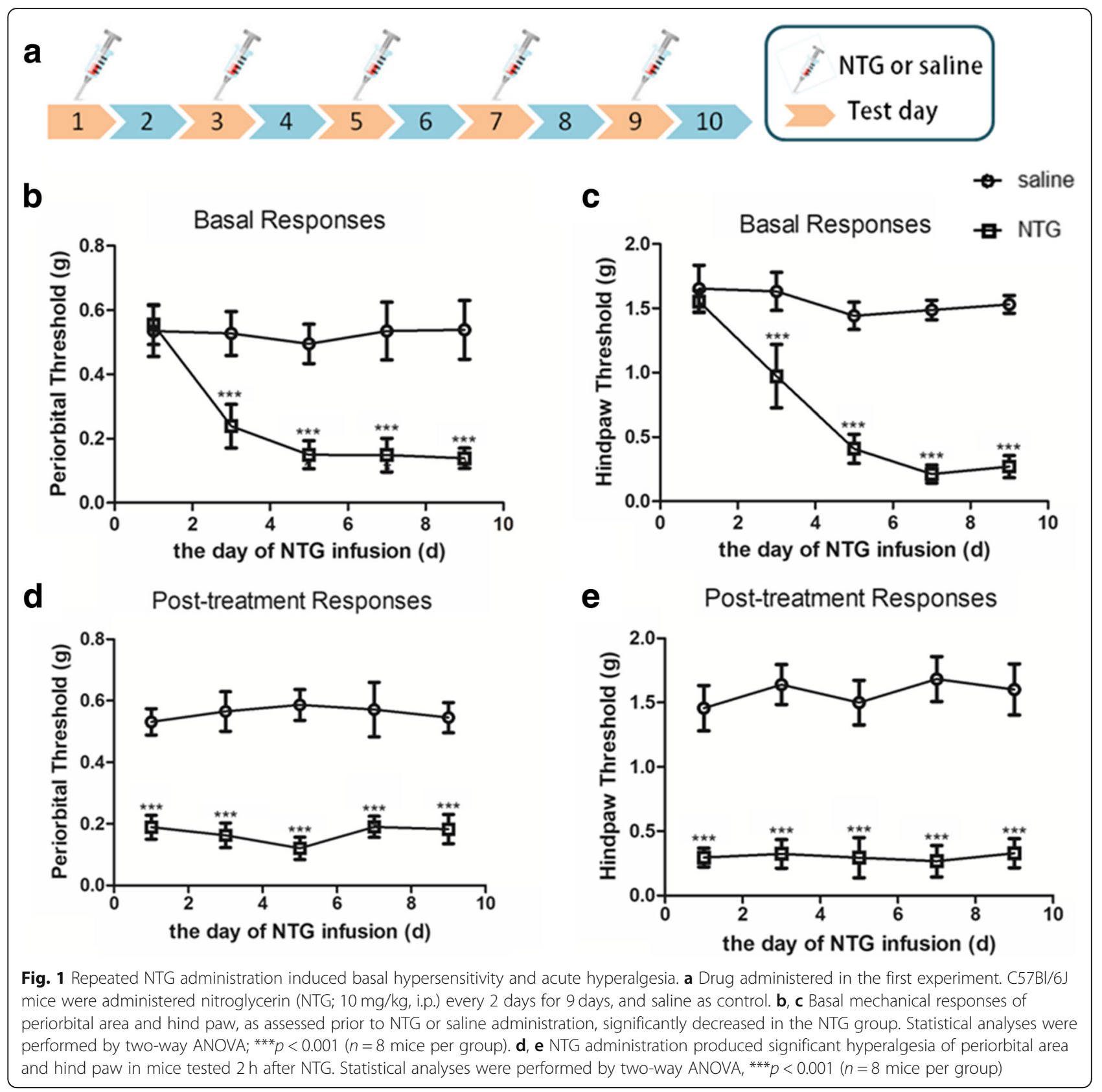

expression of NLRP3 and IL-1 $\beta$ in the TNC $(p<0.05$; Fig. $3 a-d)$. Moreover, double immunostaining indicated that NLRP3 and IL-1 $\beta$ were mainly expressed in microglia, and NLRP3 and IL-1 $\beta$ were co-localized in microglia in the TNC (Fig. 3e-g).

\section{NLRP3 inhibitor MCC950 attenuated NTG-induced} hyperalgesia and expression of CGRP, c-Fos and p-ERK in the TNC

In this experiment, mice received daily treatment with MCC950 (10 mg/kg, i.p.) or vehicle (equal volumes of PBS). On days 3, 5, 7, 9 and 11, mice were administered NTG (Fig. 4a). Daily treatment with MCC950 significantly inhibited NTG-induced basal hypersensitivity and acute hyperalgesia $(p<0.001$; Fig. 4b-e). Compared with the NTG group, preventive treatment with NLRP3 inhibitor MCC950 not only significantly decreased the protein expression levels of IL-1 $\beta$, but also decreased the protein expression levels of the CGRP, p-ERK and c-Fos in the TNC $(p<0.05$; Fig. 5).

\section{IL-1 $\beta$ antagonist IL-1 ra attenuated NTG-induced} hyperalgesia and expression of CGRP, c-Fos and p-ERK in the TNC

In this experiment, mice were received daily treatment with IL-1ra $(4 \mu \mathrm{g} / \mathrm{mouse} /$ day, I.C.V.) or vehicle (equal 
a

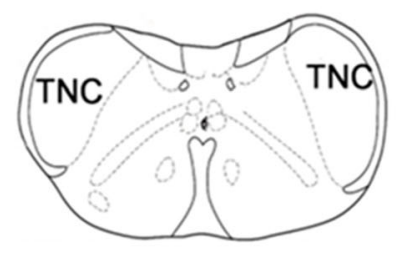

b

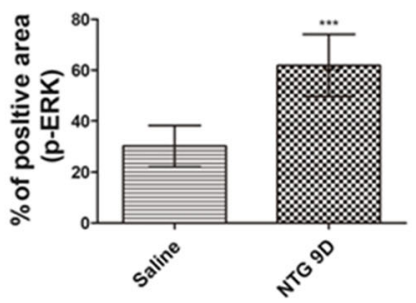

d

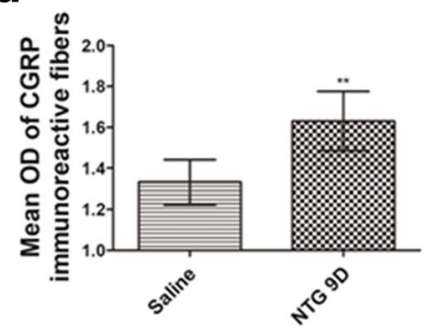

f

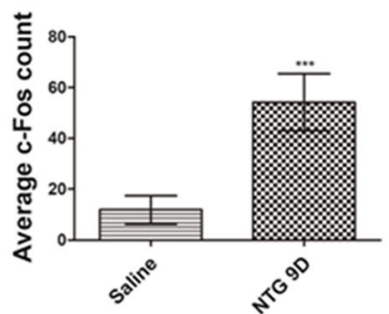

C
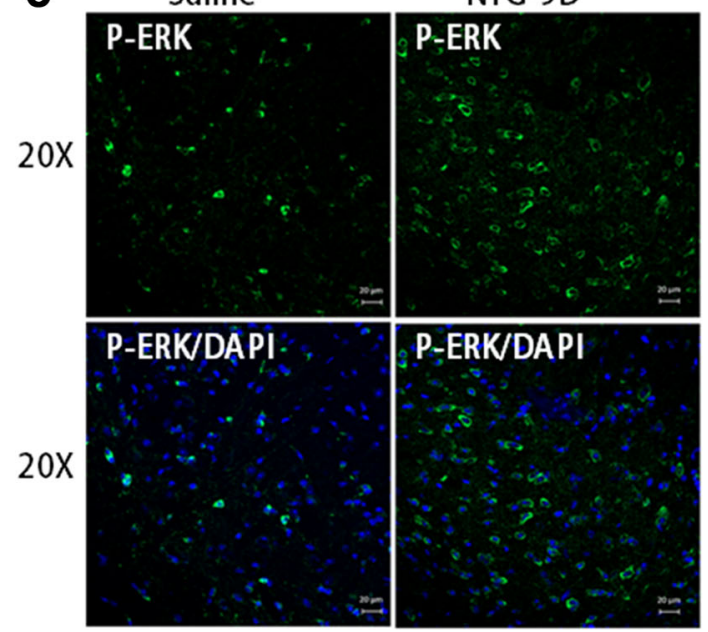

e
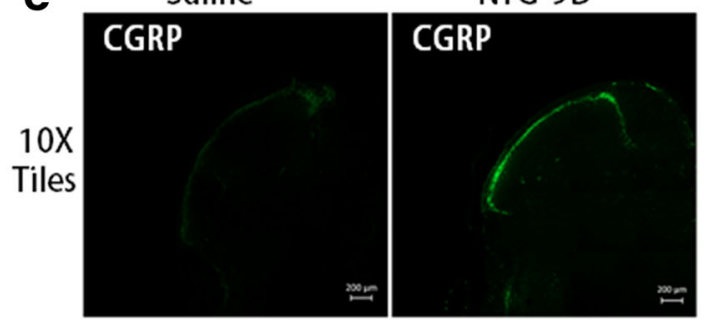

g

Saline

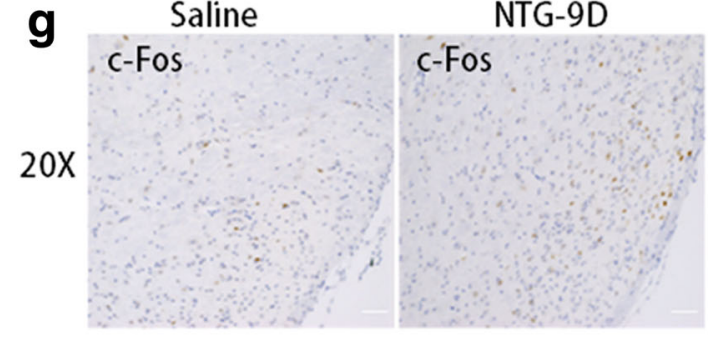

Fig. 2 Repeated NTG administration increased the expression of migraine-related proteins in the TNC, such as c-Fos, p-ERK and CGRP. a Coronal view showing the location of the TNC in the mouse brain, which is the region of interest. $\mathbf{b}$ The ratio of the p-ERK immunoreactive area to the total area of the TNC was increased in the group treated with NTG (NTG-9D group). Unpaired $t$ test, ${ }^{* * *} p<0.001$ versus the saline group ( $n=6$ mice per group). c Changes in $p$-ERK immunoreactivity in the TNC after repeated nitroglycerin (NTG) or saline administration for 9 days; $\times 20$ objective lens; scale bar, $20 \mu \mathrm{m}$. d Repeated NTG administration increased the mean OD of the CGRP-immunoreactive fibres. Unpaired $t$ test, ${ }^{* *} p<0.001$ compared with the saline group $(n=6$ mice per group). e Changes in CGRP immunoreactivity in the TNC after repeated NTG or saline administration for 9 days; $\times 10$ objective lens; scale bar, $200 \mu m$. f Quantification of the average number of c-Fos particles showed that repeated NTG administration significantly increased c-Fos expression in the TNC. Unpaired $t$ test, ${ }^{* *} p<0.001$ compared with the saline group ( $n=6$ mice per group). $\mathbf{g}$ Change in c-Fos immunoreactivity in the TNC after repeated NTG or saline administration for 9 days; $\times 20$ objective

volumes of PBS, I.C.V.) for 11 days. On days 3, 5, 7, 9 and 11, mice were administered NTG (Fig. 6a). Daily treatment with IL-1ra significantly inhibited NTG-induced basal hypersensitivity and acute hyperalgesia (Fig. 6b-e). Compared with the NTG group, preventive treatment with IL-1 $\beta$ antagonist IL-1ra significantly decreased the protein expression levels of CGRP; p-ERK and c-Fos were also significantly decreased $(p<0.001)$. However, the protein expression levels of NLRP3 were not significantly different from the NTG group $(p>0.05)$ (Fig. 7).

In addition, double immunostaining indicated that the IL- $1 \beta$ receptors, IL- $1 \mathrm{R}$ was located mainly in neurons in the TNC (Fig. 8).

\section{Discussion}

In this study, we used a published CM-associated animal model, which was established by repeated administration 

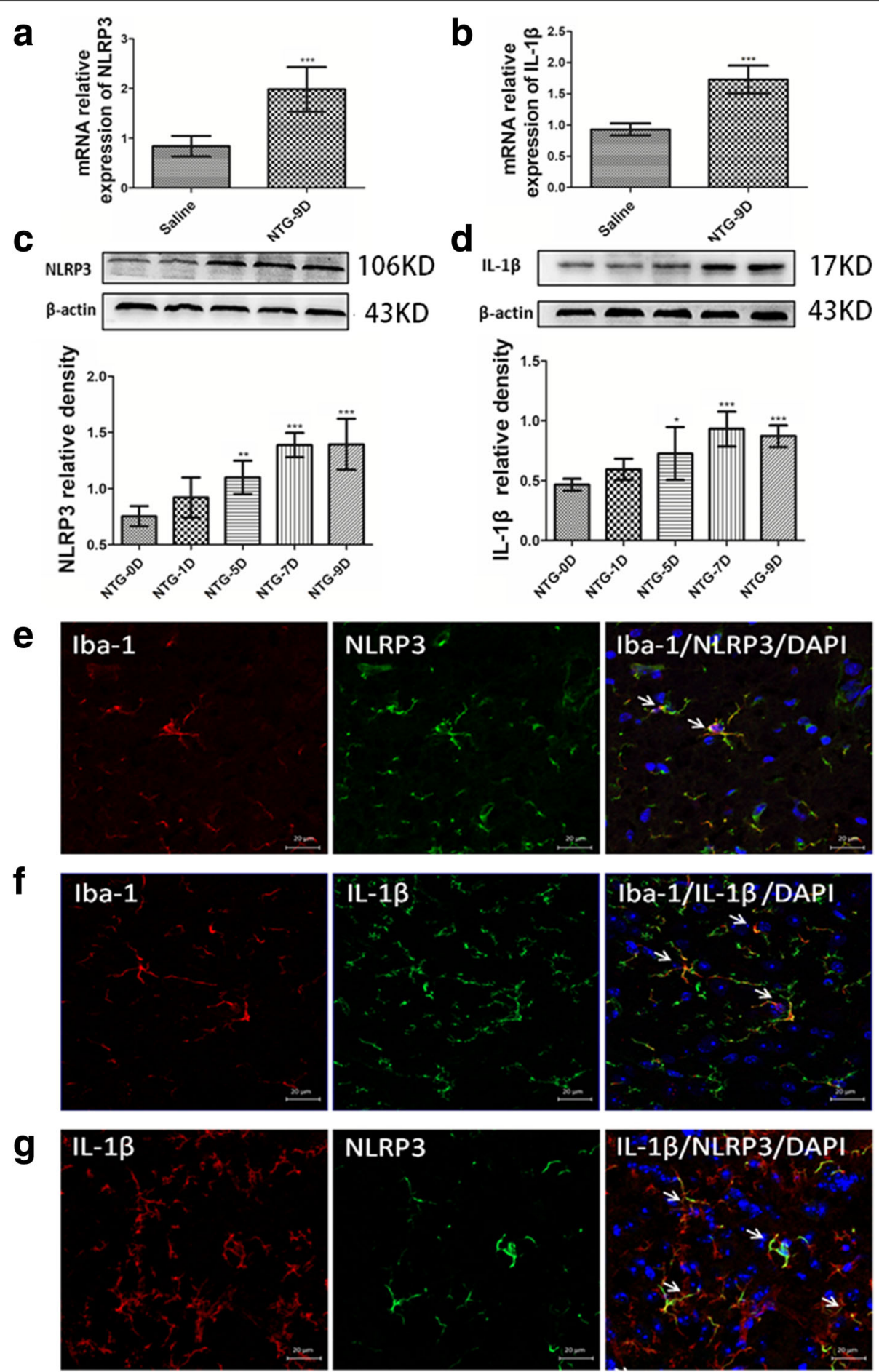

Fig. 3 Repeated NTG administration increased the expression of NLRP3 and IL-1 $\beta$ on microglia. $\mathbf{a}$, b After repeated nitroglycerin (NTG) or saline administration, NLRP3 and IL-1 $\beta$ mRNA expression analysis were tested by qRT-PCR. Unpaired $t$ test, ${ }^{* * *} p<0.001$ compared with the saline group ( $n=6$ mice per group). $\mathbf{c}$, $\mathbf{d}$ The NLRP3 and IL-1 $\beta$ protein expression were tested by western blot on different days (NTG-0D, namely before the first injection of NTG). One-way ANOVA and Tukey's multiple comparison test, ${ }^{*} p<0.05,{ }^{* *} p<0.01,{ }^{* * *} p<0.001$ versus the NTG-0D group ( $n=6$ per mice group). e Double immunofluorescence staining for Iba-1 (red) with NLRP3 (green) in the mouse TNC after repeated NTG administrations. The white arrow shows the colocalization of Iba-1 and NLRP3 proteins. $\mathbf{f}$ Double immunofluorescence staining for Iba-1 (red) and IL-1 3 (green). The white arrow shows the colocalization of IL-1 $\beta$ and Iba-1 proteins. $g$ Double immunofluorescence staining of IL-1 $\beta$ (red) and NLRP3 (green). The white arrow shows the colocalization of the IL-1 $\beta$ and NLRP3 protein. $\mathbf{e}-\mathbf{g} \times 40$ objective lens; scale bars, $20 \mu \mathrm{m}$ 


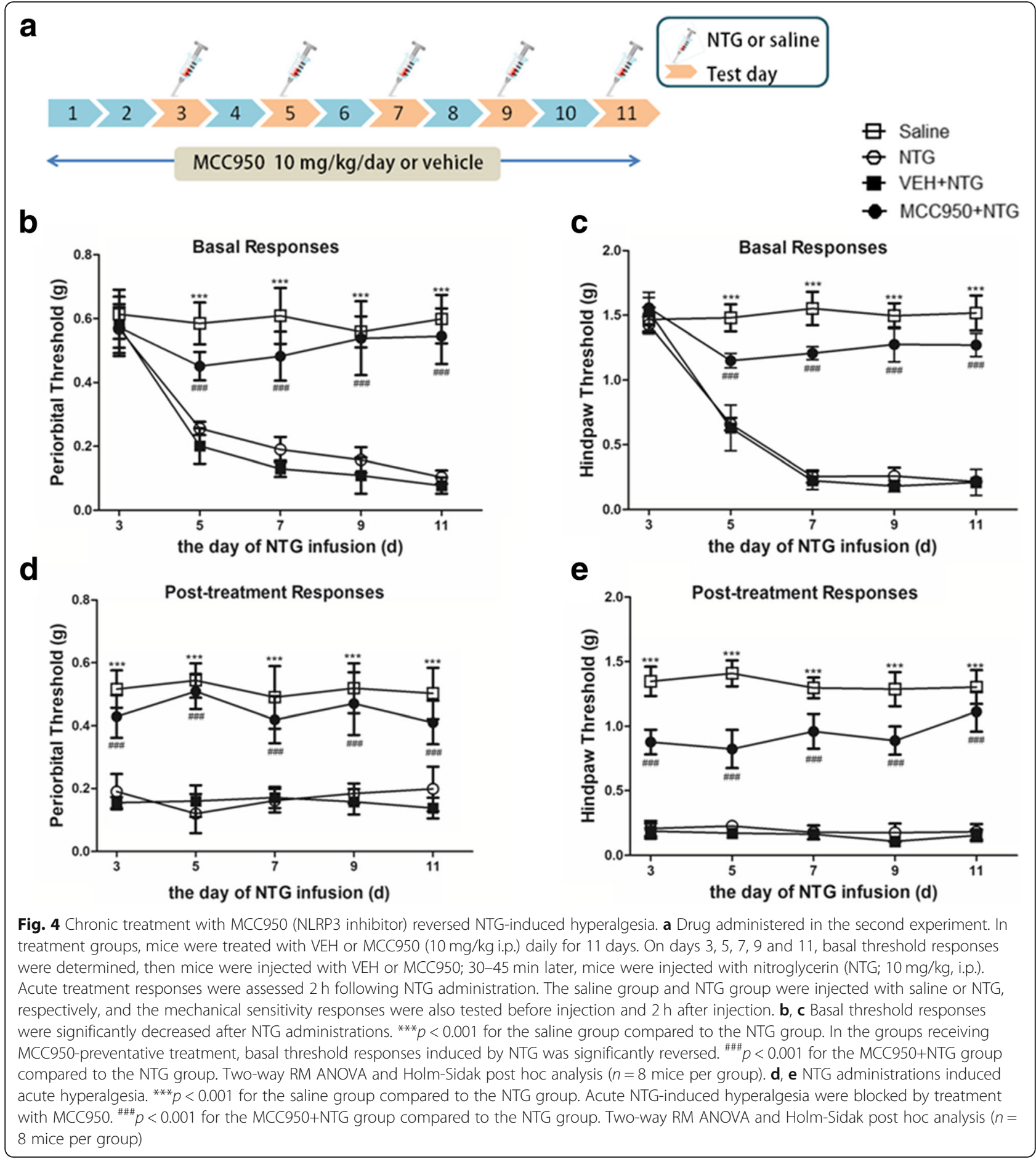

of the known human migraine trigger NTG in mice [25]. This model has been tested over the years with different migraine-related therapeutic drugs and is generally accepted as a reliable animal model of $\mathrm{CM}$-associated pain [34].

Headache is a hallmark of migraine pain; hence, we tested hypersensitivity in the specific area that represents headache response, the periorbital area, which is innervated by the trigeminal nerve V1 branch. Repeated NTG stimulation produced acute hyperalgesia and a progressive basal hypersensitivity in the area. These results are consistent with those observed in NTG-induced migraine rat models [35]. Another study in mice found repeated stimulation of high affinity nitric oxide (NO) receptor, soluble guanylyl cyclase (sGC), also caused acute hyperalgesia and baseline hypersensitivity in the periorbital area [36]. NTG-induced migraine is related 

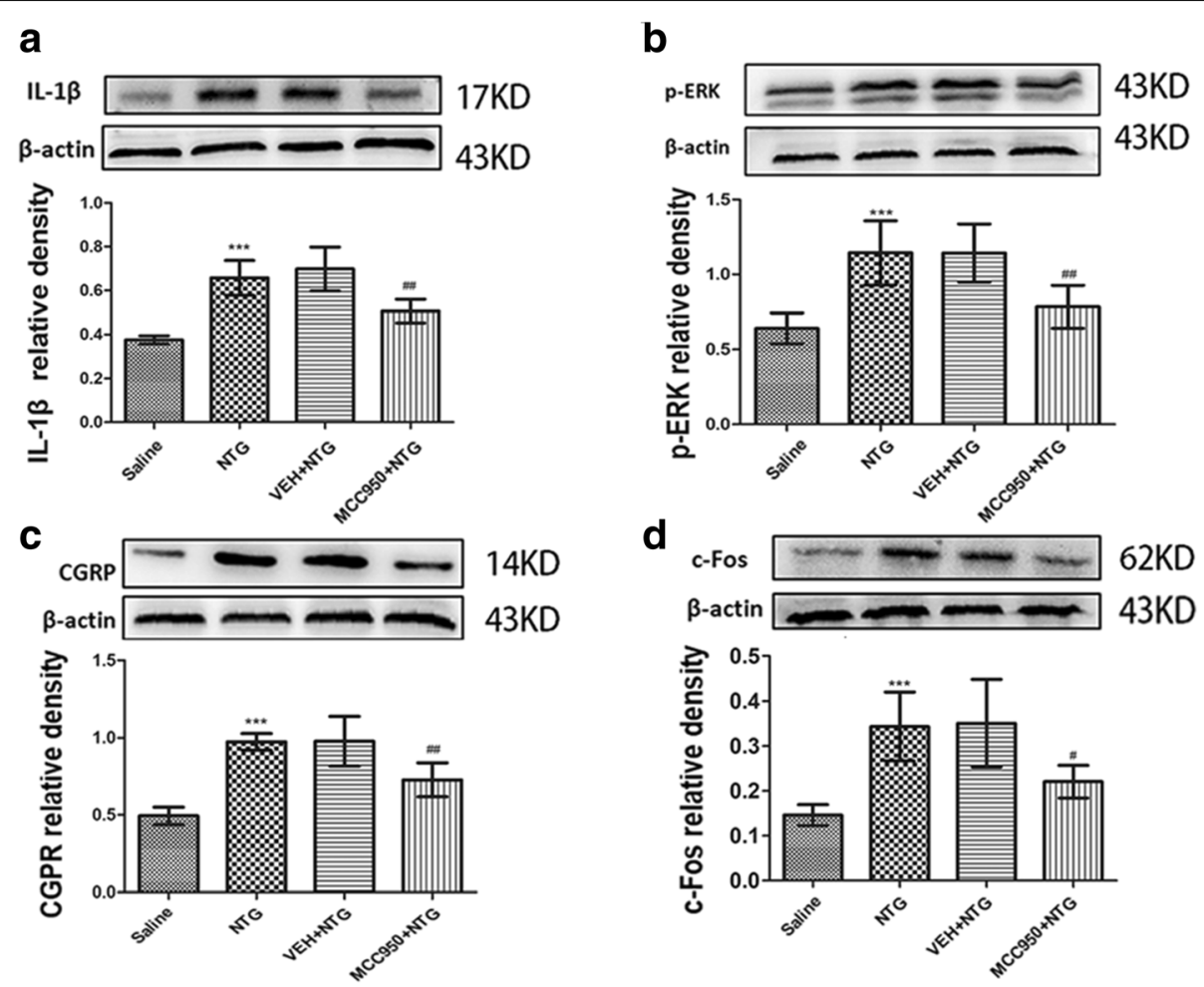

Fig. 5 Chronic treatment with MCC950 (NLRP3 inhibitor) attenuated the increased expression levels of IL-1 $\beta$, p-ERK, CGRP and c-Fos proteins. a NTG induced increase of IL-1 $\beta$ protein expression. Daily MCC950 preventative treatment decreased IL-1 $\beta$ protein expression. $\mathbf{b}-\mathbf{d}$ The protein expression of p-ERK (b), CGRP (c) and c-Fos (d) upregulated in the NTG group compared to those in the saline group; compared with NTG, daily MCC950 preventative treatment decreased these protein levels. There were no obvious differences between the NTG and NTG+PBS groups. Statistical analyses were performed by one-way ANOVA, followed by a Tukey test; ${ }^{* *} p<0.001$ vs. the saline group; ${ }^{\# \#} p<0.01,{ }^{\#} p<0.05$ vs. the NTG group ( $n=6$ mice per group)

to its metabolite $\mathrm{NO}$; therefore, the sGC stimulator VL-102 can replace NTG in the model [36]. In addition, Sol-Ji Kim reported the opposite result, which may be related to selecting different facial area; he tested whisker pads rather than periorbital area [37]. In addition to headache, CM patients may experience hyperalgesia of extracephalic areas, which is an outward manifestation of central sensitization [4, 38]. In this model, Repeated NTG stimulation also induced acute hyperalgesia and basal hypersensitivity in the hind paw, mimicking the characteristics observed in human CM patients. These results are consistent with those observed in rats or mice models that have been reported by other teams $[25,39]$.

The TNC, an important central area of the trigeminovascular system, regulates craniofacial pain. The increase in neuronal excitability in the TNC is related to the development of the central sensitization state [4]. In addition, NTG can activate neurons in the TNC area, and the increased excitability of these neurons was associated with NTG-induced hyperalgesia [35]. Therefore, we focused on the TNC as the region of interest to observe changes in biomarkers. c-Fos is a protein product of the immediate early gene and has been used extensively as a marker of neuronal activity. ERK is a member of the mitogen-activated protein kinase (MAPK) family, and phosphorylated ERK (p-ERK) is also always used as a marker of neuronal activation and central sensitization after tissue injury by noxious stimulation. Both c-Fos and p-ERK are rapidly and transiently induced following stimulation [32]. In our study, we examined the expression levels of c-Fos and p-ERK $2 \mathrm{~h}$ after the last NTG injection and found that the expression levels of both molecules were increased in the TNC. CGRP is considered an endogenous migraine generator and plays a critical role in the initiation and maintenance of migraine [40]. CGRP is synthesized and released in the superficial layer of the TNC by TG afferent fibres [41]. We detected whether the basal hypersensitivity induced by repeated NTG stimulation corresponded to an increase in CGRP expression in the TNC. Hence, we examined the CGRP expression level in the TNC $24 \mathrm{~h}$ after the last NTG injection and found that the repeated NTG stimulation increased the expression of CGRP in the TNC. The results of 


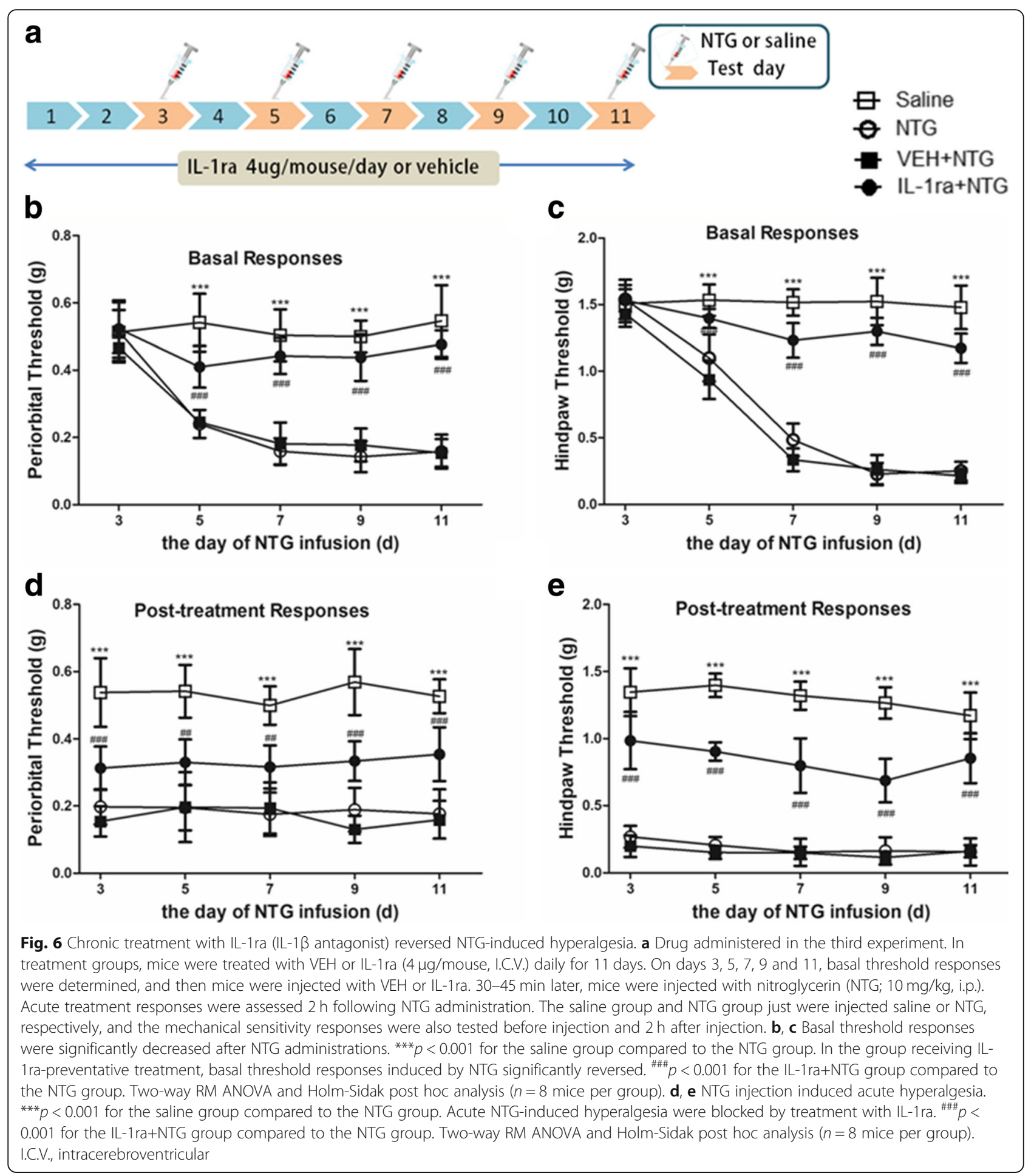

behavioural and biomarkers indicate that mice could develop a central sensitization of $\mathrm{CM}$-associated pain after repeated NTG stimulation.

Our previous studies indicated that microglia activation contributes to central sensitization in $\mathrm{CM}$-associated pain [9]. However, the specific mechanism involved in microglia is still unclear. This study found that NLRP3 was mainly expressed in microglia in the TNC. More importantly, we found that NTG-induced CM-associated pain is parallel with activation of NLRP3 inflammasomes for the first time. To further confirm the role of NLRP3 activation in migraine-associated pain, we tested the effects of the NLRP3 inhibitor MCC950 on the mice with repeated NTG stimulation. Daily MCC950 treatment not only significantly 

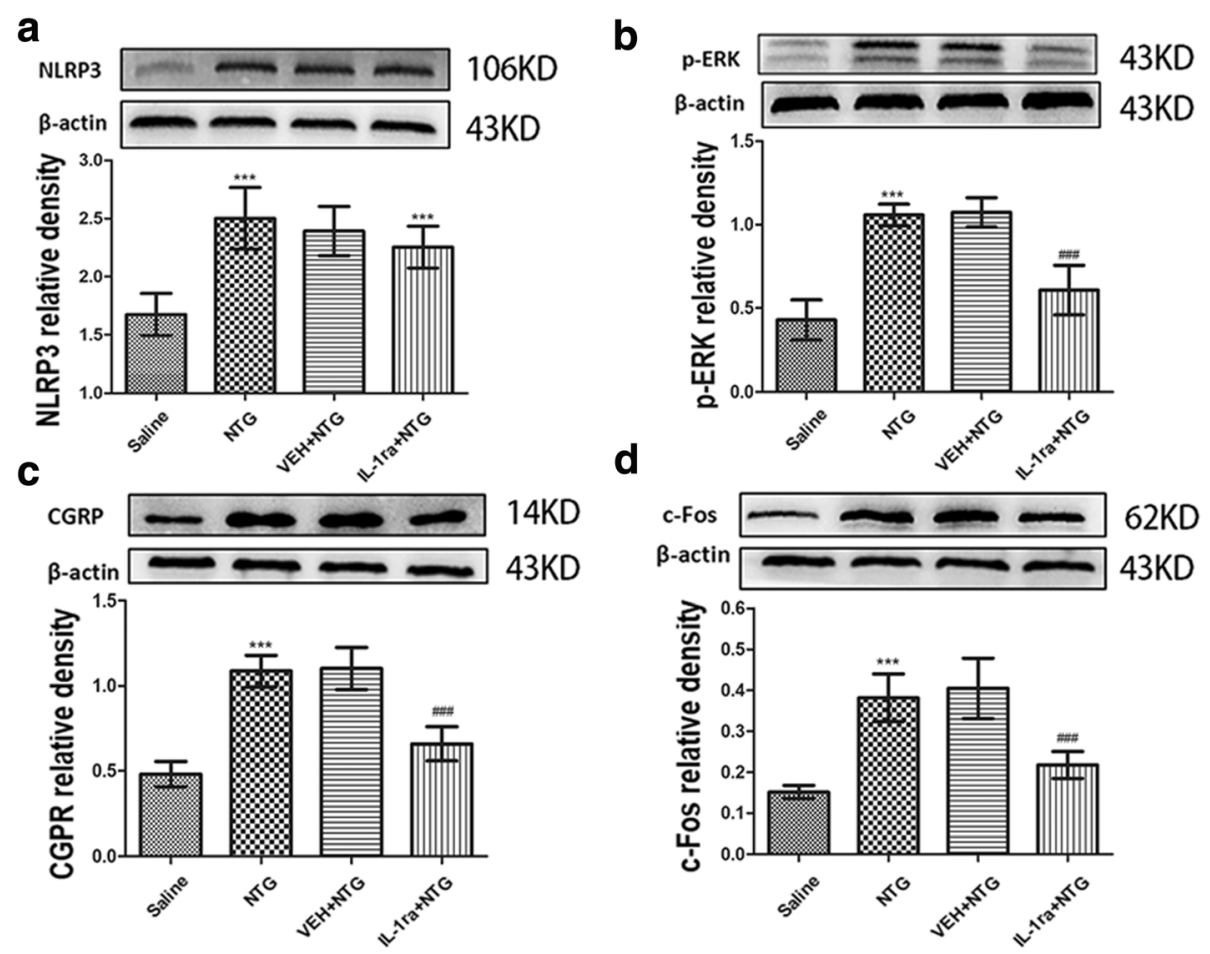

Fig. 7 Chronic treatment with IL-1ra (IL-1 $\beta$ antagonist) attenuated increased expression levels of c-Fos, p-ERK and CGRP, but not effect on NLRP3. a Nitroglycerin (NTG) induced increase of NLRP3 protein expression. But NLRP3 expression had no significant differences between the daily IL-1ra preventative treatment group and NTG group. b-d The expression of p-ERK (b), CGRP (c) and c-Fos (d) showed upregulation in the NTG group compared to those in the saline group; compared with NTG, daily IL-1 ra preventative treatment decreased these protein levels. There were no significant differences between the NTG and NTG+PBS groups. Statistical analyses were performed by one-way ANOVA, followed by a Tukey test; ${ }^{* * *} p<0.001$ vs. the saline group, ${ }^{\# \# \#} p<0.001$ vs. the NTG group ( $n=6$ mice per group)

inhibited the acute hyperalgesia and basal hypersensitivity induced by NTG but also reduced the increased expression levels of p-ERK, c-Fos and CGRP induced by NTG.

The NLRP3 promotes the processing of pro-IL-1 $\beta$ to mature IL-1 $\beta$ [13]. Studies on the peripheral mechanism of neuropathic pain indicated that IL- $1 \beta$ induce peripheral sensitization of sensory neurons and mechanical hyperalgesia [42]. Recent research found that IL-1 $\beta$ could cause plasma extravasation in the rat cerebral cortex [43]. However, whether the IL-1 $\beta$-induced plasma extravasation is related to vascular effects of migraine, there is no evidence right now. In the CNS, IL- $1 \beta$ plays a critical role in the regulation of microglia and neurons [14]. Our current results showed that the I.C.V. infusion with IL-1ra not only reverses mechanical hypersensitivity and the upregulated expression of CGRP, but also reverses upregulated expression of neuronal activation marker p-ERK and c-Fos. These data not only suggest that IL-1 $\beta$ contributes to central sensitization in $\mathrm{CM}$-associated pain mice, but also its mechanism maybe related to microglial-neuronal pathway. Double IF staining revealed that in the TNC, IL-1 $\beta$ were mainly expressed in microglia, and its receptor, IL-1R, was mainly expressed in neurons. That provides some evidence for our hypothesis.

In recent years, the relationship between NLPR3 and pain has been gradually demonstrated. In the animal model of paclitaxel-induced pain, the NLRP3 inflammasome participates in the process of peripheral sensitization [42]. Based on another study, morphine tolerance is alleviated via inhibition of NLRP3 inflammasome activation in the microglia of the spinal dorsal horn [44]. However, few studies have investigated the relationship of NLRP3 with migraines. Only one study has shown the involvement of the NLRP3 inflammasome pathway in the peripheral TG response of the rat inflammatory dural stimulation-induced model of intracranial pain [45]. However, the role of the NLRP3 inflammasome pathway in the central area remains unknown. Our research fills this gap.

Nonetheless, which danger signal induces NLRP3 activation in subjects with CM has not been established. The combination of existing studies suggests that some mechanisms mentioned below may be involved in this process. The NLRP3 inflammasome is activated by the efflux of $\mathrm{K}+$ through the open channel [46]. Purinergic receptor P2X, ligand-gated ion channel 7 receptor 


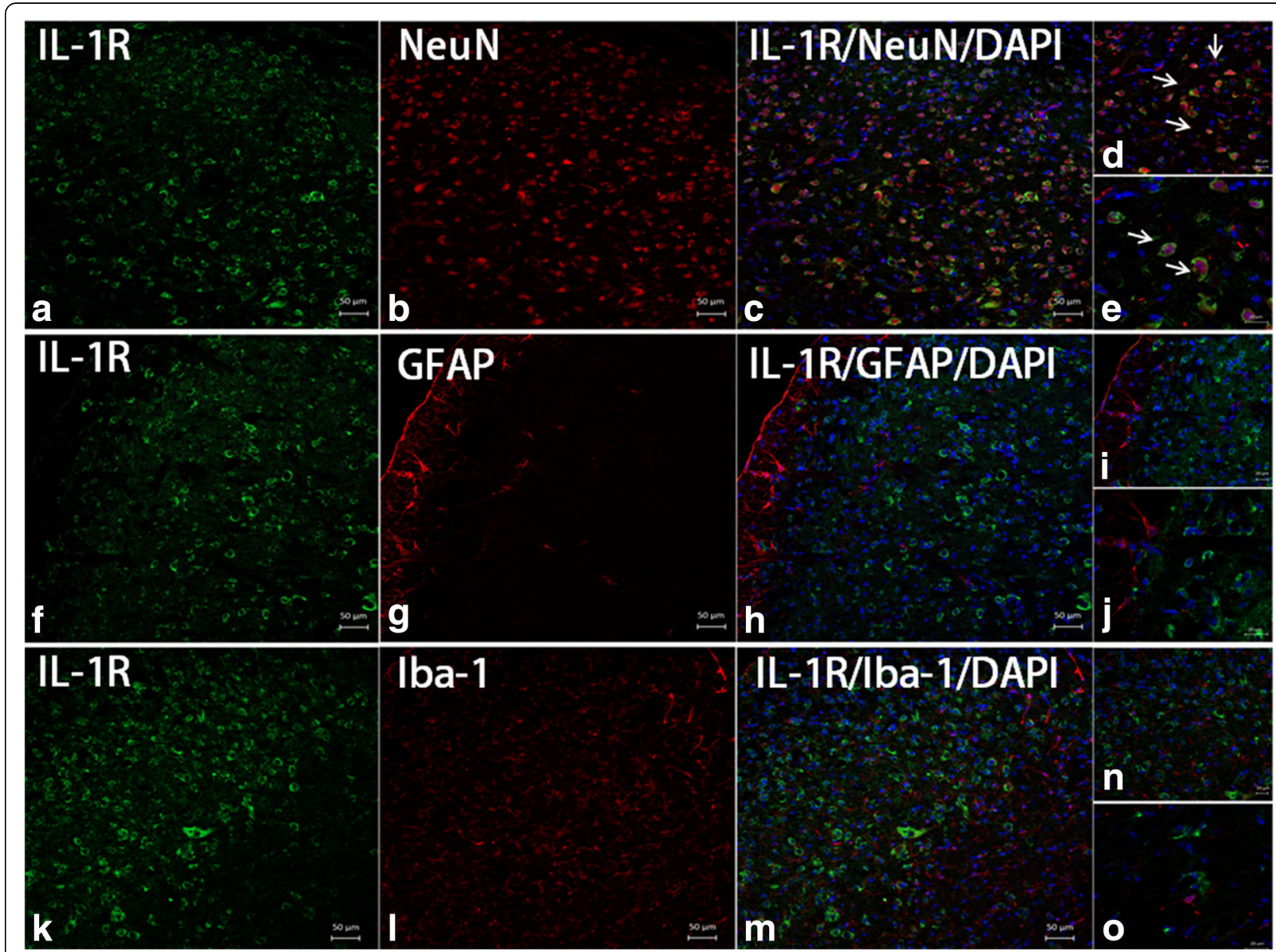

Fig. $8 \mathrm{LL}-1 \mathrm{R}$ was mainly expressed in the TNC neurons. Double immunofluorescence staining for IL-1R (green) with NeuN, a neuronal marker (red); GFAP, an astrocyte marker (red); or Iba-1, a microglial marker (red) in the mouse TNC after repeated nitroglycerin (NTG) administrations. a-e The results revealed the colocalization of IL-1R with NeuN in the TNC (white arrows). $\mathbf{f}-\mathbf{o}$ The expression of IL-1R was not detected in GFAP- or Iba-1-positive cells. a-c, $\mathbf{f}-\mathbf{h}$ and $\mathbf{k}-\mathbf{m} \times 10$ objective lens; scale bars, $50 \mu \mathrm{m}$. $\mathbf{d}$, $\mathbf{i}$ and $\mathbf{n} \times 20$ objective lens; scale bars, $20 \mu \mathrm{m}$. e, j, and $\mathbf{o} \times 40$ objective lens; scale bars, $20 \mu \mathrm{m}$

(P2X7R) is a nonselective cationic channel whose activation promotes the influx of $\mathrm{Na}+$ and $\mathrm{Ca} 2+$ into the cytosol and the concomitant efflux of $\mathrm{K}+[47]$. Moreover, P2X7R is involved in the pathophysiological process of migraine, and antagonism of P2X7R by treatment with the specific P2X7 antagonist BBG leads to the alleviation of NTG-induced thermal hypersensitivity in mice [17]. Therefore, the activation of the NLRP3 receptor in CM may be related to P2X7R. NTG is bioactivated in the body to yield NO, which may react with peroxide in vivo and produce peroxynitrite (PN), which has been associated with migraine aetiology [19]. In recent years, researchers have found that the PN pathway plays an important role in the activation mode of NLRP3 [20]. Therefore, in the NTG-induced migraine-associated pain model, PN may be produced by NTG-derived NO; activate the NLRP3 molecular platform in microglia, leading to the release of downstream inflammatory mediators; and participate in the formation of central sensitization. Mitochondrial dysfunction and ROS production also reportedly trigger NLRP3 inflammasome activation [21]. Moreover, mitochondrial dysfunction and ROS production have been proven to be related to the cause of migraine [22, 48]. In the clinic, we usually perform preventative treatment of migraine using riboflavin (vitamin B2), which supports mitochondrial function, and vitamin $C$, which modulates the effects of $\operatorname{ROS}[49,50]$.

The last topic worth mentioning is that drugs targeting the IL-1 downstream pathway of the inflammatory pathway have been developed. For example, anakinra (IL-1Ra) reportedly produces a good response to brain injury [51]. However, for some brain disorders with no damage or limited disruption of the blood-brain barrier, such as migraine, anakinra may not easily access the brain. The NLRP3 inflammasome is an available therapeutic target. 
To date, the most advanced NLRP3 small molecule inhibitor is MCC950 [29]. Our results confirm that MCC950 is protective in the NTG-induced migraine-associated pain model.

\section{Conclusions}

Our study demonstrates that the expression of the NLRP3 inflammasome was upregulated in the migraine-associated pain mouse model that was induced by recurrent NTG stimulation. The increase in NLRP3 expression levels can activate IL-1 $\beta$ signalling. Blockade of NLRP3 and IL-1 $\beta$ both improved hyperalgesia and inhibited the increase in these biomarkers related to central sensitization of $\mathrm{CM}$ such as p-ERK, c-Fos and CGRP in the TNC (Fig. 9).

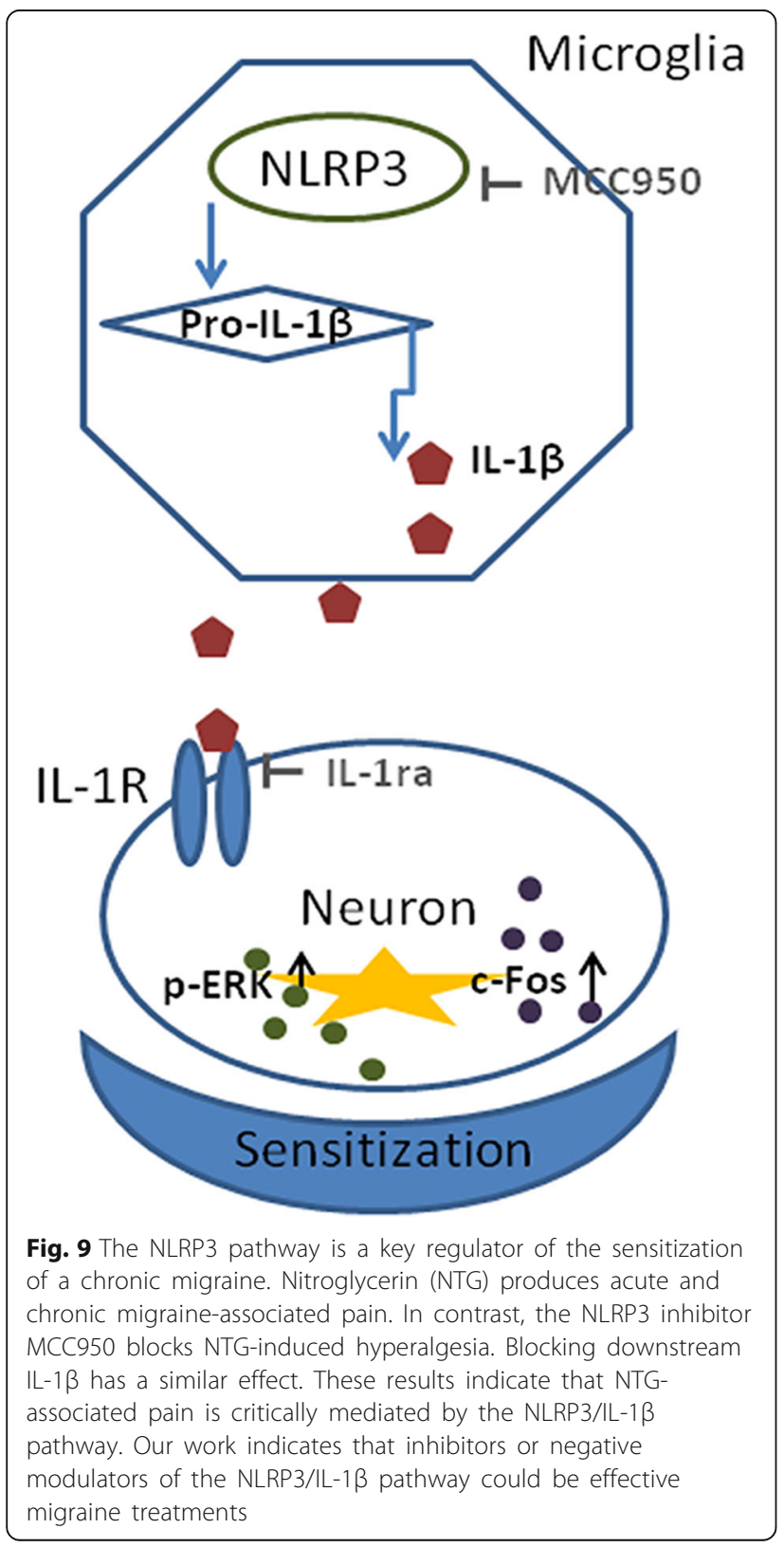

Together, these results provide new information regarding the physiopathology of CM. The NLRP3 inflammasome plausibly constitutes a target for the control of CM-associated pain, and its inhibition may represent a new therapeutic rationale and approach for migraine treatment.

\section{Additional files}

Additional file 1: Figure S1. Scheme of the experimental design. (a) Experiment 1: Mice were randomly divided into the following groups: the nitroglycerin (NTG) group and saline group. (b) Experiment 2: Mice were randomly divided into the following groups: NTG, saline, NTG+VEH and NTG+MCC950 groups. Experiment 3: Mice were randomly divided into the following groups: NTG, saline, NTG+VEH, NTG+IL-1 ra groups. VEH, vehicle; h, hour. (TIF 5765 kb)

Additional file 2: Figure S2. Repeated NTG administration increased the expression of microglial markers in the mouse TNC. (a) Coronal view showing the location of the TNC in the mouse brain. (b) Immunoreactive quantitative analysis of Iba-1 (microglial marker). Repeated nitroglycerin (NTG) or saline administered for 9 days. Compared to the saline group, the NTG group showed an increased ratio of the Iba-1-immunoreactive area to the total area of the TNC. Analyzed at $\times 10$ images. Unpaired $t$ test, ${ }^{* * *} p<0.001$ versus the saline group ( $n=5$ mice per group). $(c, d)$ Immunostaining of the TNC for Iba-1 in the saline group and NTG group; nuclear staining was performed with DAPI (middle column). (c) $\times 10$ objective lens; scale bar, $50 \mu \mathrm{m}$; (d) $\times 40$ objective lens; scale bar, $20 \mu \mathrm{m}$. (TIF 3402 kb)

\section{Abbreviations}

BCA: Bicinchoninic acid; BLAST: Basic local alignment search tool; CGRP: Calcitonin gene-related peptide; CM: Chronic migraine; CNS: Central nervous system; DAB: Diaminobenzidine; DAPI: 4,6-Diamidino-2-phenylindole; GAPDH: Glyceraldehyde 3-phosphate dehydrogenase; GFAP: Glial fibrillary acidic protein; I.C.V.: Intracerebroventricular; i.p.: Intraperitoneal;

IF: Immunofluorescence; IgG: Immunoglobulin; IH: Immunohistochemistry; IL1R: IL-1 receptor; IL-1ra: IL-1 receptor antagonist; IL-1 $\beta$ : Interleukin-1 $\beta$; MAPK: Mitogen-activated protein kinase; $n$ : Number in each experiment or group; NeuN: Neuronal nuclei; NLRP3: NOD-like receptor protein 3; NO: Nitric oxide; NTG: Nitroglycerin; OD: Optical density; P2X7: Purinergic receptor P2X, ligand-gated ion channel 7; PBS: Phosphate-buffered saline; $\mathrm{p}$ ERK: Phosphorylated extracellular signal-regulated kinase; PFA: Paraformaldehyde; PN: Peroxynitrite; qRT-PCR: Quantitative real-time polymerase chain reaction; ROS: Reactive oxygen species; SD: Standard deviation; SDS PAGE: Sodium dodecyl sulfate polyacrylamide gel electrophoresis; sGC: Soluble guanylyl cyclase; TBS: Tris-buffered saline; TG: Trigeminal ganglion; TNC: Trigeminal nucleus caudalis; VEH: Vehicle

\section{Acknowledgements}

Not applicable.

\section{Funding}

This work was supported by the research grants from Chongqing Yuzhong District Science and Technology Plan Project (No: 20170106) and the National Natural Science Foundation of China (No: 81671092).

\section{Availability of data and materials}

The data used in this article are available if necessary.

\section{Authors' contributions}

WH conceived the study, performed most of the experiments, analyzed the data and wrote the manuscript. TL assisted with some WB experiments and provided advice on experimental design. QP and SZ participated in some behavioural experiments. DZ and GQ assisted with some IF experiments. YZ provided advice on histological experiments. JZ and LC supervised the experimental design. JZ revised the manuscript. All of the authors read and approved the final manuscript. 


\section{Ethics approval and consent to participate}

Our manuscript data were collected from animals and the study was approved by the Ethics Committee for Animal Experimentation of Chongqing Medical University (Document number SYXK (YU) 2012-0001).

\section{Consent for publication}

Not applicable.

\section{Competing interests}

The authors declare that they have no competing interests.

\section{Publisher's Note}

Springer Nature remains neutral with regard to jurisdictional claims in published maps and institutional affiliations.

\section{Author details}

'Department of Neurology, The First Affiliated Hospital of Chongqing Medical University, 1st You Yi Road, Yu Zhong District, Chongqing 400016, People's Republic of China. ${ }^{2}$ Laboratory Research Center, The First Affiliated Hospital of Chongqing Medical University, 1st You Yi Road, Yu Zhong District, Chongqing 400016, People's Republic of China.

\section{Received: 23 December 2018 Accepted: 22 March 2019}

\section{Published online: 10 April 2019}

\section{References}

1. Stovner L, Hagen K, Jensen R, Katsarava Z, Lipton R, Scher A, Steiner T, Zwart JA. The global burden of headache: a documentation of headache prevalence and disability worldwide. Cephalalgia. 2007;27:193-210.

2. Natoli JL, Manack A, Dean B, Butler Q, Turkel CC, Stovner L, Lipton RB. Global prevalence of chronic migraine: a systematic review. Cephalalgia. 2010;30:599-609.

3. Tfelt-Hansen $\mathrm{P}$, Olesen J. Taking the negative view of current migraine treatments: the unmet needs. CNS Drugs. 2012;26:375-82.

4. Bernstein C, Burstein R. Sensitization of the trigeminovascular pathway: perspective and implications to migraine pathophysiology. J Clin Neurol. 2012:8:89-99.

5. Latremoliere A, Woolf CJ. Central sensitization: a generator of pain hypersensitivity by central neural plasticity. J Pain. 2009;10:895-926.

6. Xie YF, Zhang S, Chiang CY, Hu JW, Dostrovsky JO, Sessle BJ. Involvement of glia in central sensitization in trigeminal subnucleus caudalis (medullary dorsal horn). Brain Behav Immun. 2007;21:634-41.

7. Peng J, Gu N, Zhou L, EU B, Murugan M, Gan WB, Wu L. Microglia and monocytes synergistically promote the transition from acute to chronic pain after nerve injury. Nat Commun. 2016;7:12029.

8. Bartley J. Could glial activation be a factor in migraine? Med Hypotheses. 2009; $72: 255-7$

9. Long T, He W, Pan Q, Zhang S, Zhang Y, Liu C, Liu Q, Qin G, Chen L, Zhou J. Microglia P2X4 receptor contributes to central sensitization following recurrent nitroglycerin stimulation. J Neuroinflammation. 2018:15:245.

10. Sarchielli P, Alberti A, Baldi A, Coppola F, Rossi C, Pierguidi L, Floridi A, Calabresi P. Proinflammatory cytokines, adhesion molecules, and lymphocyte integrin expression in the internal jugular blood of migraine patients without aura assessed ictally. Headache. 2006:46:200-7.

11. De Corato A, Lisi L, Capuano A, Tringali G, Tramutola A, Navarra P, Dello RC. Trigeminal satellite cells express functional calcitonin gene-related peptide receptors, whose activation enhances interleukin-1 beta pro-inflammatory effects. J Neuroimmunol. 2011;237:39-46.

12. Liu X, Quan N. Microglia and CNS interleukin-1: beyond immunological concepts. Front Neurol. 2018;9:8.

13. Martinon F, Burns K, Tschopp J. The inflammasome: a molecular platform triggering activation of inflammatory caspases and processing of proll-beta. Mol Cell. 2002;10:417-26.

14. Pan Y, Chen XY, Zhang QY, Kong LD. Microglial NLRP3 inflammasome activation mediates IL-1beta-related inflammation in prefrontal cortex of depressive rats. Brain Behav Immun. 2014;41:90-100.

15. Ma Q, Chen S, Hu Q, Feng H, Zhang JH, Tang J. NLRP3 inflammasome contributes to inflammation after intracerebral hemorrhage. Ann Neurol. 2014;75:209-19.

16. Gris D, Ye Z, locca HA, Wen H, Craven RR, Gris P, Huang M, Schneider M, Miller SD, Ting JP. NLRP3 plays a critical role in the development of experimental autoimmune encephalomyelitis by mediating Th1 and Th17 responses. J Immunol. 2010;185:974-81.

17. Goloncser F, Sperlagh B. Effect of genetic deletion and pharmacological antagonism of P2X7 receptors in a mouse animal model of migraine. J Headache Pain. 2014;15:24.

18. Rajamaki K, Nordstrom T, Nurmi K, Akerman KE, Kovanen PT, Oorni K, Eklund KK. Extracellular acidosis is a novel danger signal alerting innate immunity via the NLRP3 inflammasome. J Biol Chem. 2013;288:13410-9.

19. Yilmaz $G$, Surer $H$, Inan $L E$, Coskun $O$, Yucel D. Increased nitrosative and oxidative stress in platelets of migraine patients. Tohoku J Exp Med. 2007;211:23-30.

20. Bellezza I, Grottelli S, Costanzi E, Scarpelli P, Pigna E, Morozzi G, Mezzasoma L, Peirce MJ, Moresi V, Adamo S, Minelli A. Peroxynitrite activates the NLRP3 inflammasome cascade in SOD1(G93A) mouse model of amyotrophic lateral sclerosis. Mol Neurobiol. 2018;55:2350-61.

21. He Y, Hara H, Nunez G. Mechanism and regulation of NLRP3 inflammasome activation. Trends Biochem Sci. 2016;41:1012-21.

22. Sparaco M, Feleppa M, Lipton RB, Rapoport AM, Bigal ME. Mitochondrial dysfunction and migraine: evidence and hypotheses. Cephalalgia. 2006;26:361-72.

23. Abais JM, Xia M, Zhang Y, Boini KM, Li PL. Redox regulation of NLRP3 inflammasomes: ROS as trigger or effector? Antioxid Redox Signal. 2015;22:1111-29.

24. Zimmermann M. Ethical guidelines for investigations of experimental pain in conscious animals. Pain. 1983;16:109-10.

25. Pradhan AA, Smith ML, McGuire B, Tarash I, Evans CJ, Charles A. Characterization of a novel model of chronic migraine. Pain. 2014;155:269-74.

26. Abraham J, Jang S, Godbout JP, Chen J, Kelley KW, Dantzer R, Johnson RW. Aging sensitizes mice to behavioral deficits induced by central HIV-1 gp120. Neurobiol Aging. 2008;29:614-21.

27. Abraham J, Johnson RW. Central inhibition of interleukin-1 beta ameliorates sickness behavior in aged mice. Brain Behav Immun. 2009:23:396-401.

28. Dempsey C, Rubio AA, Bryson KJ, Finucane O, Larkin C, Mills EL, Robertson A, Cooper MA, O'Neill L, Lynch MA. Inhibiting the NLRP3 inflammasome with MCC950 promotes non-phlogistic clearance of amyloid-beta and cognitive function in APP/PS1 mice. Brain Behav Immun. 2017;61:306-16.

29. Coll RC, Robertson AAB, Chae JJ, Higgins SC, Muñoz-Planillo R, Inserra MC, Vetter I, Dungan LS, Monks BG, Stutz A, et al. A small-molecule inhibitor of the NLRP3 inflammasome for the treatment of inflammatory diseases. Nat Med. 2015;21:248-55.

30. Livak KJ, Schmittgen TD. Analysis of relative gene expression data using real-time quantitative PCR and the 2(-Delta Delta C(T)) method. Methods. 2001:25:402-8.

31. Pinskiy V, Tolpygo AS, Jones J, Weber K, Franciotti N, Mitra PP. A low-cost technique to cryo-protect and freeze rodent brains, precisely aligned to stereotaxic coordinates for whole-brain cryosectioning. J Neurosci Methods. 2013:218:206-13.

32. Gao YJ, Ji RR. c-Fos and pERK, which is a better marker for neuronal activation and central sensitization after noxious stimulation and tissue injury? Open Pain J. 2009;2:11-7.

33. Riesco N, Cernuda-Morollon E, Pascual J. Neuropeptides as a marker for chronic headache. Curr Pain Headache R. 2017;21(18).

34. Tipton AF, Tarash I, McGuire B, Charles A, Pradhan AA. The effects of acute and preventive migraine therapies in a mouse model of chronic migraine. Cephalalgia. 2016;36:1048-56.

35. Farajdokht F, Mohaddes G, Shanehbandi D, Karimi P, Babri S. Ghrelin attenuated hyperalgesia induced by chronic nitroglycerin: CGRP and TRPV1 as targets for migraine management. Cephalalgia. 2018;38:1716-30.

36. Ben AM, Tipton AF, Bertels Z, Gandhi R, Moye LS, Novack M, Bennett BM, Wang $Y$, Litosh $V$, Lee $S H$, et al. Soluble guanylyl cyclase is a critical regulator of migraine-associated pain. Cephalalgia. 2018;38:1471-84.

37. Kim SJ, Yeo JH, Yoon SY, Kwon SG, Lee JH, Beitz AJ, Roh DH. Differential development of facial and hind paw allodynia in a nitroglycerin-induced mouse model of chronic migraine: role of capsaicin sensitive primary afferents. Biol Pharm Bull. 2018:41:172-81.

38. Burstein R. Deconstructing migraine headache into peripheral and central sensitization. Pain. 2001;89:107-10.

39. Greco R, Demartini C, Zanaboni AM, Redavide E, Pampalone S, Toldi J, Fulop F, Blandini F, Nappi G, Sandrini G, et al. Effects of kynurenic acid analogue 1 (KYNA-A1) in nitroglycerin-induced hyperalgesia: targets and anti-migraine mechanisms. Cephalalgia. 2017;37:1272-84.

40. Cady RJ, Glenn JR, Smith KM, Durham PL. Calcitonin gene-related peptide promotes cellular changes in trigeminal neurons and glia implicated in peripheral and central sensitization. Mol Pain. 2011;7:94. 
41. Iyengar S, Ossipov MH, Johnson KW. The role of calcitonin gene-related peptide in peripheral and central pain mechanisms including migraine. Pain. 2017;158:543-59.

42. Jia M, Wu C, Gao F, Xiang H, Sun N, Peng P, Li J, Yuan X, Li H, Meng X, et al. Activation of NLRP3 inflammasome in peripheral nerve contributes to paclitaxel-induced neuropathic pain. Mol Pain. 2017;13:2071443716.

43. Richter F, Eitner A, Leuchtweis J, Bauer R, Lehmenkuhler A, Schaible H. Effects of interleukin-1 $\beta$ on cortical spreading depolarization and cerebral vasculature. J Cerebr Blood F Met. 2017;37:1791-802.

44. Cai Y, Kong $H$, Pan YB, Jiang $L$, Pan XX, Hu L, Qian YN, Jiang CY, Liu WT. Procyanidins alleviates morphine tolerance by inhibiting activation of NLRP3 inflammasome in microglia. J Neuroinflammation. 2016;13:53.

45. Chen L, Li X, Huang L, Wu Q, Chen L, Wan Q. Chemical stimulation of the intracranial dura activates NALP3 inflammasome in trigeminal ganglia neurons. Brain Res. 2014;1566:1-11.

46. Munoz-Planillo R, Kuffa P, Martinez-Colon G, Smith BL, Rajendiran TM, Nunez G. K(+) efflux is the common trigger of NLRP3 inflammasome activation by bacterial toxins and particulate matter. Immunity. 2013;38:1142-53.

47. Buisman HP, Steinberg TH, Fischbarg J, Silverstein SC, Vogelzang SA, Ince C, Ypey DL, Leijh PC. Extracellular ATP induces a large nonselective conductance in macrophage plasma membranes. Proc Natl Acad Sci U S A. 1988:85:7988-92

48. Raddant AC, Russo AF. Reactive oxygen species induce procalcitonin expression in trigeminal ganglia glia. Headache. 2014;54:472-84.

49. Colombo B, Saraceno L, Comi G. Riboflavin and migraine: the bridge over troubled mitochondria. Neurol Sci. 2014:35(Suppl 1):141-4.

50. Shaik MM, Gan SH. Vitamin supplementation as possible prophylactic treatment against migraine with aura and menstrual migraine. Biomed Res Int. 2015;2015:469529.

51. Brough D, Rothwell NJ, Allan SM. Interleukin-1 as a pharmacological target in acute brain injury. Exp Physiol. 2015;100:1488-94.

Ready to submit your research? Choose BMC and benefit from:

- fast, convenient online submission

- thorough peer review by experienced researchers in your field

- rapid publication on acceptance

- support for research data, including large and complex data types

- gold Open Access which fosters wider collaboration and increased citations

- maximum visibility for your research: over $100 \mathrm{M}$ website views per year

At $\mathrm{BMC}$, research is always in progress.

Learn more biomedcentral.com/submissions 